General Letters in Mathematics (GLM) 1(1) (2016) 45-52
General Letters in Mathematics (GLM)
Website: http://www.sciencereflection.com/general-letters-in-mathematics/
Reflection

\title{
Non Linear Free-Surface Flow Over a Submerged Obstacle
}

\author{
Abdelkrim Merzougui $^{1}$, Abdelkader Laiadi $^{2}$

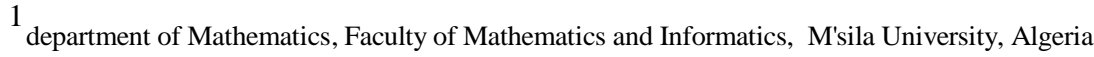 \\ 1shamdadz@yahoo.fr \\ 2 \\ Biskra University, department of Mathematics, Algeria \\ 2laiadhi_a@yahoo.fr
}

\begin{abstract}
Free-surface flow over a triangular obstacle is considered. The fluid is assumed to be inviscid, incompressible and the flow is assumed to be steady and irrotational. Both gravity and surface tension are included in the dynamic boundary condition. Far upstream, the flow is assumed to be uniform. The problem is solved numerically using a boundary integral equation method. The problem is solved by first deriving integro-differential equations. These equations are discretized and the resulting nonlinear algebraic equations are solved by Newton method. When surface tension and gravity are included, there are two additional parameters in the problem known as the Weber number and Froude number.
\end{abstract}

\section{Indexing terms/Keywords}

Free surface flow, potential flow, Weber number, surface tension, Froude number.

\section{SUBJECT CLASSIFICATION}

MSC[2010]: 76B10, 76C05, 76M25

\section{Introduction}

Research has been done in this area for more than 150 years and it still continues today. Over these years problems have been solved including only gravity g, or surface tension $\mathrm{T}$ and also problems that consider both. The reason for neglecting or including such properties is to isolate what effect surface tension or gravity has on a free surface.

This paper considers the problem of steady free-surface of a two-dimensional, irrotational, inviscid and incompressible flow. The problem is first formulated as an integral equation for the unknown shapes of the free surfaces. This equation is then discretized and the resulting algebraic equations are solved by Newton's method. Later on, we found numerical solutions of free-surface flows over a triangular obstacle with the effects of surface tension and the gravity. The problem is formulated in section 2 , the numerical procedure is described in section 4 and the results are discussed in section 5. 


\section{Mathematical Formulation}

We consider the fluid flow over a triangular obstacle. The fluid is inviscid and incompressible and the flow is irrotational. The flow domain is bounded above by a free surface $E G F$ and below by a horizontal bottom and the triangle $B C D$ with angle $\gamma$ where $0<\gamma<\frac{\pi}{2}$. We introduce Cartesian coordinates $(x, y)$ (see Figure 1).

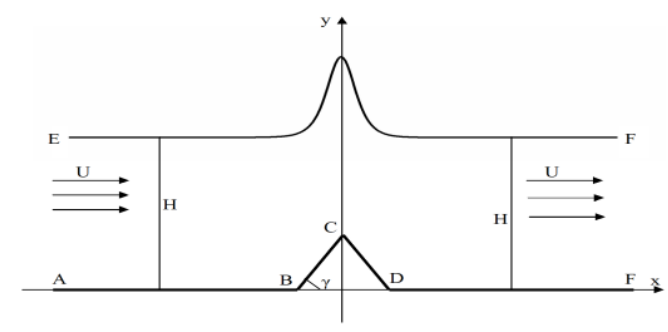

Fig 1: Sketch of a free-surface flow in physical coordinates $(x, y)$

As $x \rightarrow \infty$, the flow is assumed to approach a uniform stream with constant velocity $U$ and constant length $H$. It is convenient to define dimensionless variables by taking $U$ as the unit velocity and $H$ as the unit length. The dimensionless parameters in the problem are the Froude number $F=\frac{U}{\sqrt{g H}}$ and the inverse Weber number $\delta=\frac{T}{\rho U^{2} H}$. Here $T$ is the surface tension $g$ the gravity and $\rho$ is the fluid density. We introduce the complex potential function and the complex velocity

$$
\begin{aligned}
& f(x, y)=\phi(x, y)+i \psi(x, y) \\
& \omega=\frac{\partial f}{\partial z}=u-i v
\end{aligned}
$$

Where $u$ and $v$ are the velocity components in the $x$ and $y$ directions and $z=x+i y$. We choose $\psi=0$ along the free surface $E F$. Then $\psi=-1$ and $-\infty<\phi<+\infty$ along the bottom ABCDF. The flow configuration in the $f$-plane is sketched in figure 2 .

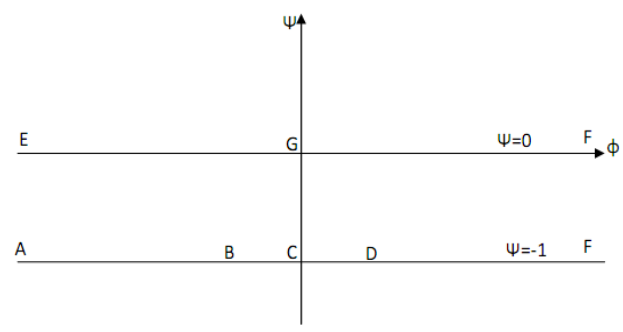

Fig 2: Sketch of the complex potential $f$-plane 
The mathematical problem can be formulated in terms of the potential function $\phi$ satisfying the Laplace's equation

$$
\Delta \phi=0 \text { in the fluiddomain }
$$

The effects of gravity and the surface tension are considered. Bernoulli's equation gives

$$
\frac{1}{2}\left(u^{* 2}+v^{* 2}\right)+\frac{p^{*}}{\rho^{*}}+g^{*} y^{*}=C
$$

Using the capillary Laplace's equation defined by:

$$
p^{*}-p_{0}=\frac{T}{R}=K T
$$

where $K=\frac{1}{R}$ is the curvature, $\mathrm{p}^{*}$ is the fluid pressure, $\mathrm{p}_{0}$ is the atmospheric pressure, $\rho^{*}$ is the fluid density and $g$ is the gravitational constant. Substituting (2.4) into (2.3) and in terms of the dimensionless variables, Bernoulli's equation on the free surface becomes:

$$
\frac{1}{2}\left(u^{2}+v^{2}\right)+\delta K+\frac{1}{F^{2}}(y-1)=\frac{1}{2}
$$

The kinematic boundary conditions are

$$
\left\{\begin{array}{l}
v=0 \text { on } \psi=-1,-\infty<\phi<\phi_{B}, \phi_{D}<\phi<+\infty \\
v=u \tan |\gamma| \text { on } \psi=-1, \phi_{B}<\phi<\phi_{C}, \phi_{C}<\phi<\phi_{D}
\end{array}\right.
$$

Now we reformulate the problem as an integral equation. We define the function $\tau-i \theta$ by:

$$
\omega=u-i v=e^{\tau-i \theta}
$$

We map the flow domain onto the upper half of the $\zeta$-plane by the transformation

$$
\zeta=\alpha+i \beta=e^{-\pi f}=e^{-\pi \phi}(\cos \pi \psi-\sin \pi \psi)
$$

as shown in figure 3 .

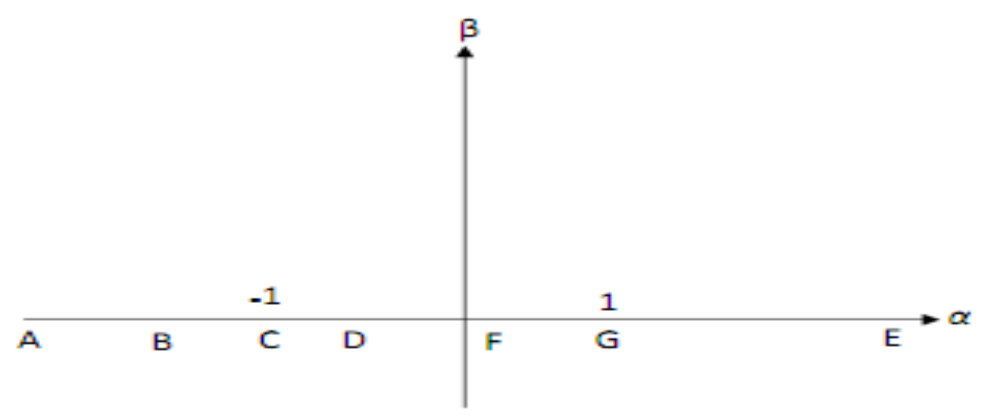

Fig 3: the upper-half $\zeta$-plane. 
The velocity terms, first, become:

$$
u^{2}+v^{2}=e^{2 \tau}
$$

Secondly, the curvature $K$ of a streamline, in terms of $\theta$, is

$$
K=-e^{\tau}\left|\frac{\partial \theta}{\partial \phi}\right|
$$

Substituting (2.9) and (2.10) into (2.5), gives the final form of Bernoulli's equation that is needed for the numerical calculation. This is

$$
\frac{1}{2} e^{2 \tau}-\delta e^{\tau}\left|\frac{\partial \theta}{\partial \phi}\right|+\frac{1}{F^{2}}(y-1)=\frac{1}{2} \text { on } E F
$$

Using (2.8), the kinematic boundary conditions (2.6) in the $\zeta$-plane, become

$$
\left\{\begin{array}{lll}
\theta=0 & \text { for }-\infty<\phi<\phi_{B}, & \phi_{D}<\phi<+\infty, \\
\theta=\gamma & \text { for } \phi_{B}<\phi<\phi_{C} & \text { and } \psi=-1 \\
\theta=-\gamma & \text { for } \quad \phi_{C}<\phi<\phi_{D} & \text { and } \psi=-1 \\
\theta=\text { unknown } & \text { for }-\infty<\phi<+\infty & \text { and } \psi=0
\end{array}\right.
$$

\section{Boundary integral techniques}

Next we apply the Cauchy integral formula to the function $\tau-i \theta$ in the complex $\zeta$-plane. We choose a contour consisting of the real axis and a half-circle of arbitrary large radius in the upper half-plane. After taking the real part we obtain

$$
\tau\left(\alpha_{0}\right)=-\frac{1}{\pi} \int_{-\infty}^{+\infty} \frac{\theta(\alpha)}{\alpha-\alpha_{0}} d \alpha
$$

Where $\tau\left(\alpha_{0}\right)=\tau\left(\alpha_{0}, 0\right)$ and $\theta(\alpha)=\theta(\alpha, 0)$ are used to simplify the notation. Using the boundary conditions (2.12), the integral in (3.1) to give

$$
\tau\left(\alpha_{0}\right)=-\frac{1}{\pi}\left[\begin{array}{l}
\int_{-\infty}^{\alpha_{B}} \frac{\theta(\alpha)}{\alpha-\alpha_{0}} d \alpha+\int_{\alpha_{B}}^{\alpha_{C}} \frac{\theta(\alpha)}{\alpha-\alpha_{0}} d \alpha+\int_{\alpha_{C}}^{\alpha_{D}} \frac{\theta(\alpha)}{\alpha-\alpha_{0}} d \alpha \\
\int_{\alpha_{D}}^{\alpha_{F}} \frac{\theta(\alpha)}{\alpha-\alpha_{0}} d \alpha+\int_{0}^{+\infty} \frac{\theta(\alpha)}{\alpha-\alpha_{0}} d \alpha
\end{array}\right]
$$

Using the conditions (2.12), equation (3.2) can be simplified as:

$$
\tau\left(\alpha_{0}\right)=-\frac{\gamma}{\pi} \log \left|\frac{\alpha_{C}-\alpha_{0}}{\alpha_{B}-\alpha_{0}}\right|+\frac{\gamma}{\pi} \log \left|\frac{\alpha_{D}-\alpha_{0}}{\alpha_{C}-\alpha_{0}}\right|-\frac{1}{\pi} \int_{0}^{+\infty} \frac{\theta(\alpha)}{\alpha-\alpha_{0}} d \alpha
$$

This equation holds along the free surface and so using (2.7), with $\psi=0$ gives:

$$
\alpha=e^{-\pi \phi}
$$


Substituting (3.4) into (3.3), yields:

$$
\tau^{\prime}\left(\phi_{0}\right)=-\frac{\gamma}{\pi} \log \left|\frac{e^{-\pi \phi_{C}}+e^{-\pi \phi_{0}}}{e^{-\pi \phi_{B}}+e^{-\pi \phi_{0}}}\right|+\frac{\gamma}{\pi} \log \left|\frac{e^{-\pi \phi_{D}}+e^{-\pi \phi_{0}}}{e^{-\pi \phi_{C}}+e^{-\pi \phi_{0}}}\right|+\int_{-\infty}^{\phi} \frac{\theta^{\prime}(\phi) e^{-\pi \phi}}{e^{-\pi \phi}-e^{-\pi \phi_{0}}} d \phi
$$

For $-\infty<\phi<+\infty$

where $\tau^{\prime}\left(\phi_{0}\right)=\tau(\phi, 0)$ and $\theta^{\prime}(\phi)=\theta(\phi, 0)$. Equation (2.11) is now rewritten in terms of $\tau^{\prime}$ and $\theta^{\prime}$ as:

$$
\frac{1}{2} e^{2 \tau^{\prime}}-\delta e^{\tau^{\prime}}\left|\frac{\partial \theta^{\prime}}{\partial \phi}\right|+\frac{1}{F^{2}}(y-1)=\frac{1}{2} \text { on } E F
$$

Next we evaluate the values of $y$ on the free surfaces by using (2.9) and integrating the identity

$$
\frac{d z}{d f}=\omega^{-1}
$$

This gives:

$$
y(\alpha)=1-\frac{1}{\pi} \int_{0}^{\alpha} \frac{e^{-\tau\left(\alpha_{0}\right)} \sin \theta\left(\alpha_{0}\right)}{\alpha_{0}} d \alpha_{0} \text { for } 0<\alpha<+\infty
$$

By using (3.4), we rewrite (3.8) as:

$$
y^{\prime}(\phi)=1+\int_{-\infty}^{\phi} e^{-\tau^{\prime}\left(\phi_{0}\right)} \sin \theta^{\prime}\left(\phi_{0}\right) d \phi_{0} \quad \text { for }-\infty<\phi<+\infty
$$

By substituting (3.5) and (3.9) into (3.6), an integro-differential equation is created and this is solved numerically in the following section.

\section{Numerical Procedure}

In Section 3, a nonlinear integro-differential equation was derived. In this Section, the numerical procedure used to calculate solutions to this equation will be discussed. The expression (3.5) is used to calculate $\tau$ along the free surface. It is required to have points, $\phi_{0}$, along the free surface at which $\tau$ values can be evaluated. This is done by creating an equally spaced mesh, in the potential function, over the region that relates to the free surface. Let

$$
\phi_{I}=\left[\frac{-(N-1)}{2}+(I-1)\right] \Delta, \quad I=1, \ldots, N,-\infty<\phi<+\infty
$$

where $\Delta>0$, is the uniform increment between consecutive mesh points. The integral (3.5) is evaluated by the trapezoidal rule, with a summation over $\phi_{I}$ such that $\phi_{0}$ is the midpoint of one sub-interval, defined as follows: 


$$
\phi_{M}=\frac{\phi_{I+1}+\phi_{I}}{2}, I=1, \ldots, N-1
$$

Firstly, (3.5) is rewritten as:

$$
\tau_{M}=\tau^{\prime}\left(\phi_{M}\right)=-\frac{\gamma}{\pi} \log \left|\frac{e^{-\pi \phi_{C}}+e^{-\pi \phi_{M}}}{e^{-\pi \phi_{B}}+e^{-\pi \phi_{M}}}\right|+\frac{\gamma}{\pi} \log \left|\frac{e^{-\pi \phi_{D}}+e^{-\pi \phi_{M}}}{e^{-\pi \phi_{C}}+e^{-\pi \phi_{M}}}\right|+\int_{-\infty}^{+\infty} \frac{\theta^{\prime}(\phi) e^{-\pi \phi}}{e^{-\pi \phi}-e^{-\pi \phi_{M}}} d \phi
$$

where $\tau_{M}=\tau^{\prime}\left(\phi_{M}\right)$ has been introduced to ease notation. Then, using the trapezoidal rule gives:

$$
\tau_{M}=-\frac{\gamma}{\pi} \log \left|\frac{e^{-\pi \phi_{C}}+e^{-\pi \phi_{M}}}{e^{-\pi \phi_{B}}+e^{-\pi \phi_{M}}}\right|+\frac{\gamma}{\pi} \log \left|\frac{e^{-\pi \phi_{D}}+e^{-\pi \phi_{M}}}{e^{-\pi \phi_{C}}+e^{-\pi \phi_{M}}}\right|+\sum_{j=1}^{N} \frac{\theta_{j} e^{-\pi \phi_{j}} \Delta w_{j}}{e^{-\pi \phi_{j}}-e^{-\pi \phi_{M}}}
$$

where $I=1, \ldots, N-1$ and $w_{j}$ is the weighting function such that

$$
w_{j}=\left\{\begin{array}{l}
\frac{1}{2}: j=1, N \\
1: \text { otherwise }
\end{array}\right.
$$

and $\theta_{j}=\theta^{\prime}\left(\phi_{j}\right)$

By substituting (4.4) into (3.6), evaluated at the mesh points (4.1), a system of $N$ nonlinear equations in $N$ unknowns is obtained. These are $\theta_{I}$ for $I=1, \ldots, N$. We evaluate $y_{I}=y^{\prime}\left(\phi_{I}\right)$ by applying the trapezoidal rule to (4.4) and by using (3.7). This yields

$$
\left\{\begin{array}{l}
y_{1}=1 \\
y_{I+1}=y_{I}+\Delta e^{-\tau_{M}} \sin \theta_{M}, \quad I=1, \ldots, N-1
\end{array}\right.
$$

and

$$
x_{I+1}=x_{I}+\Delta e^{-\tau_{M}} \sin \theta_{M}, I=1, \ldots, N-1
$$

Here $\theta_{M}=\frac{\theta_{I+1}+\theta_{I}}{2}$. We now satisfy (3.6) at the midpoints (4.2). This yields $N$ nonlinear algebraic equations for the $N$ unknowns $\theta_{I}, I=1, \ldots, N$. The derivative $\frac{\partial \theta^{\prime}}{\partial \phi}$, at the mesh points (4.1), is approximated by a finite difference, whereby

$$
\frac{\partial \theta^{\prime}}{\partial \phi}=\frac{\theta_{I+1}-\theta_{I}}{\Delta}, I=1, \ldots, N-1
$$

For a given value of $\delta$ and $F$, this system of $N$ equations with $N$ unknowns is solved by Newton's method. 


\section{Discussion of the Result}

We used the numerical scheme described in section 4 to compute solutions for various values of $F$ and $\delta$. Most of the calculations were performed with $\mathrm{N}=300, \Delta=0.1$, and $\gamma=\frac{\pi}{4}$. We also calculated solutions with smaller values of $\Delta$ and larger values of $\mathrm{N}$ and checked that the results presented here are independent of these parameters within graphical accuracy. The results shown in Figures 4are examples of free surface profiles calculated using the numerical scheme discussed in Section 4.
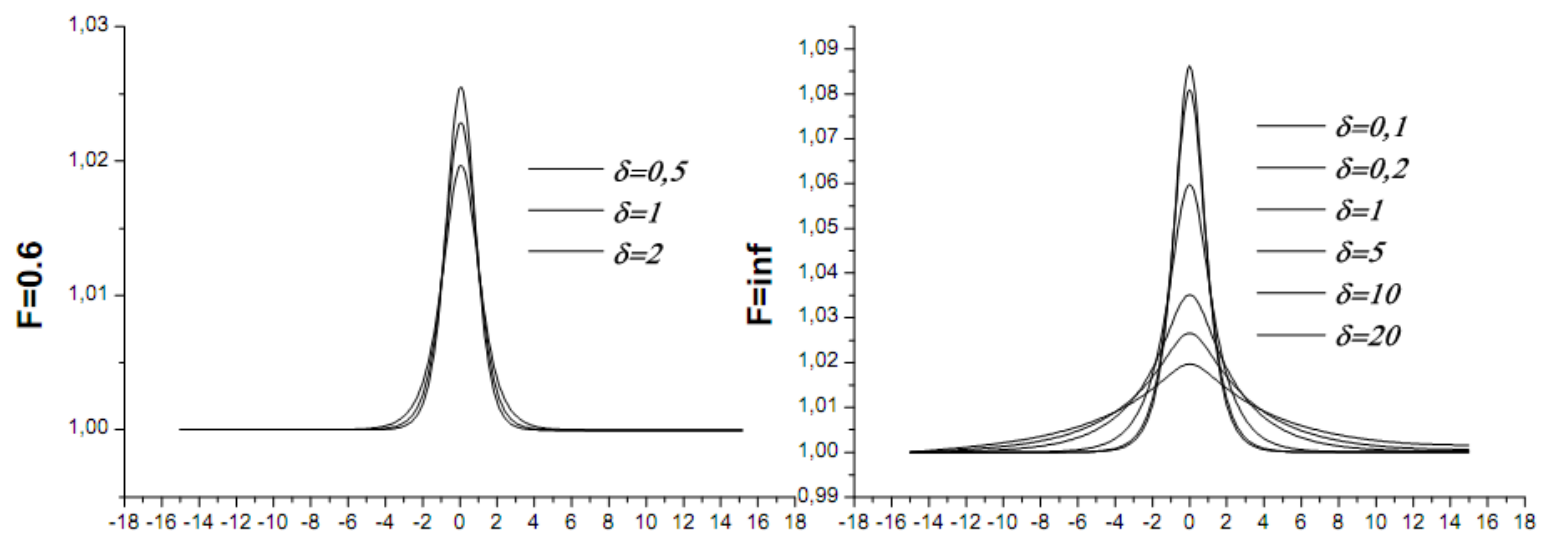

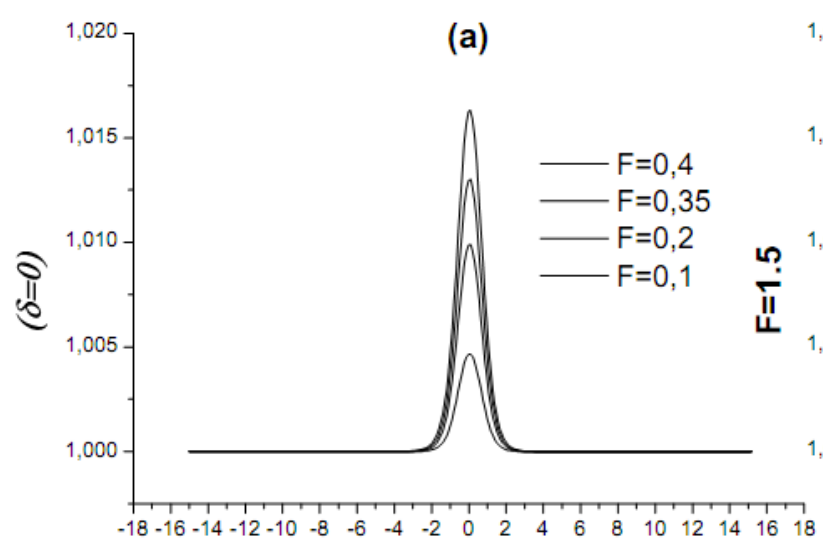

(c) (b)

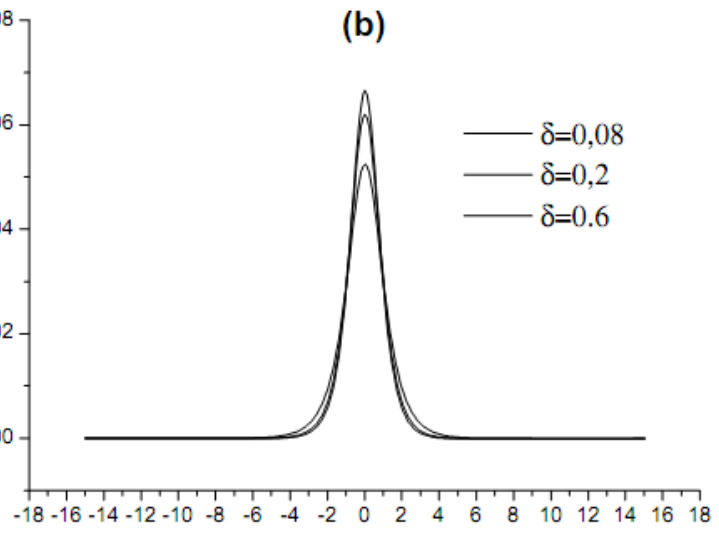

(d)

Fig 4 a) Subcritical flows for $\mathrm{F}=0.6$ and $\delta=0.5,1,2$ (increasing amplitude) b) Free surface profiles for $\mathrm{F}=\infty$ and various $\delta$ c) Free surface profiles for $\delta=0$ and various $\mathrm{F}$ d) Supercritical flows for $\mathrm{F}=1.5$ and various $\delta$

\section{References}

[1] Dias, F., Vanden-Broeck, J.-M. 2002. "Generalized critical free-surface flows” J. Eng. Math., 42, 291-301.

[2] Forbes, L.K., Schwartz, L.W. 1982. "Free-surface flow over a semi-circular obstruction in a channel" J. Fluid. Mech., $114,299-314$. 
[3] Forbes, L.K. 1983. "Free-surface flow over a semi-circular obstruction, including the influence of gravity and surface tension" J. Fluid. Mech., 127, 283-297.

[4] Hocking G. C, Vanden-Broeck, J.M. 1997. "Draining of a fluid of finite depth into a vertical slot". Appl. Math. Modeling ,vol 21, 643-649.

[5] Vanden-Broeck, J. M. 1997. "Numerical calculations of the free-surface flow under a sluice gate". J. Fluid. Mech. vol. 330, pp. 339-347. 